\title{
REFUTATION OF A CLAIM MADE BY FEJES TÓTH ON THE ACCURACY OF SURFACE MESHES
}

\author{
GERT VEGTER ${ }^{1}$ and MATHIJS WINTRAECKEN ${ }^{2}$ \\ 1 Johann Bernoulli Institute for Mathematics and Computer Science, Nijenborgh 9, 9747 AG \\ Groningen, P. O. Box 407, 9700 AK Groningen, The Netherlands, \\ e-mail: g.vegter@rug.nl \\ 2 IST Austria, Am Campus 1, 3400 Klosterneuburg, Austria \\ e-mail: mathijs.wintraecken@ist.ac.at
}

Communicated by J. Pach

(Received November 18, 2019; accepted January 23, 2020)

\begin{abstract}
Fejes Tóth [3] studied approximations of smooth surfaces in three-space by piecewise flat triangular meshes with a given number of vertices on the surface that are optimal with respect to Hausdorff distance. He proves that this Hausdorff distance decreases inversely proportional with the number of vertices of the approximating mesh if the surface is convex. He also claims that this Hausdorff distance is inversely proportional to the square of the number of vertices for a specific non-convex surface, namely a one-sheeted hyperboloid of revolution bounded by two congruent circles. We refute this claim, and show that the asymptotic behavior of the Hausdorff distance is linear, that is the same as for convex surfaces.
\end{abstract}

\section{Introduction}

In [3] Fejes Tóth introduced inscribed triangulations approximating convex surfaces in $\mathbb{R}^{3}$ optimally and the approximation parameter $A_{2}$ (Approximierbarkeit). By a triangulation we shall mean a geometric realization of a simplicial complex in Euclidean space homeomorphic to the surface, that is piecewise linear in ambient space. From now on we take a simplicial complex to mean the geometric realization.

2010 Mathematics Subject Classification. Primary 68U05.

Key words and phrases. Hausdorff distance, Approximation, Fejes Tóth, surface meshes. 
Optimal triangulations with $m$ vertices are triangulations which minimize the Hausdorff distance between the surface and the simplicial complex when this simplicial complex ranges over the space of triangulations with $m$ vertices. We always assume that the vertices lie on the surface.

The Hausdorff distance between two subsets $X$ and $Y$ in Euclidean space is defined as:

$$
d_{H}(X, Y)=\max \left\{\sup _{x \in X} \inf _{y \in Y}|x-y|, \sup _{y \in Y} \inf _{x \in X}|x-y|\right\},
$$

where $|x-y|$ denotes the standard Euclidean distance of $x$ and $y$. The onesided Hausdorff distance from $X$ to $Y$ is given by

$$
d_{H}^{o}(X, Y)=\sup _{x \in X} \inf _{y \in Y}|x-y| .
$$

The inverse of the asymptotic value of the product of the number of vertices and the Hausdorff distance is referred to as the approximation parameter (Approximierbarkeit) $A_{2}$.

Fejes Tóth [3] gave the expression

$$
\frac{1}{A_{2}}=\lim _{m \rightarrow \infty} d_{H}\left(\Sigma, T_{m}\right) m \geq \frac{1}{\sqrt{27}} \int \sqrt{K} \mathrm{~d} A,
$$

where $K$ is the Gaussian curvature, for the approximation parameter for convex surfaces in three dimensional Euclidean space. We refer to $[1,4,5]$ for an introduction to geometry including the Gaussian curvature.

Fejes Tóth also claimed that the approximation of ruled surfaces embedded in three dimensional Euclidean space would be entirely different from the approximation of convex surfaces. In Section 12 of Chapter 5 of [3] he states the following:

Let $\Sigma$ be the one-sheeted hyperboloid bounded by two congruent circles $A$ and $B$. We inscribe $A$ and $B$ by regular $m$-polygons $A_{1} \ldots A_{m}$ and $B_{1} \ldots B_{m}$ respectively, so that $A_{1} B_{1}, \ldots, A_{m} B_{m}$ lie on the hyperboloid. The polyhedron-like surface $T_{2 m}$ is best described by its faces

$$
A_{1} A_{2} B_{1}, \ldots, A_{m} A_{1} B_{m} \quad \text { and } \quad B_{1} B_{2} A_{2}, \ldots, B_{m} B_{1} A_{1} .
$$

The deviation $d_{H}\left(T_{2 m}, \Sigma\right)$ is determined by the deviation of the $m$-polygon $A_{1} \ldots A_{m}$ from the circle $A$, this implies that the order of magnitude of the deviation is $1 / \mathrm{m}^{2}$ and not $1 / \mathrm{m}$.

Unfortunately this is incorrect. In fact we shall show that the order of magnitude of $d_{H}\left(\Sigma, T_{m}\right)$ is $1 / m$, like in the convex case. Moreover in this 


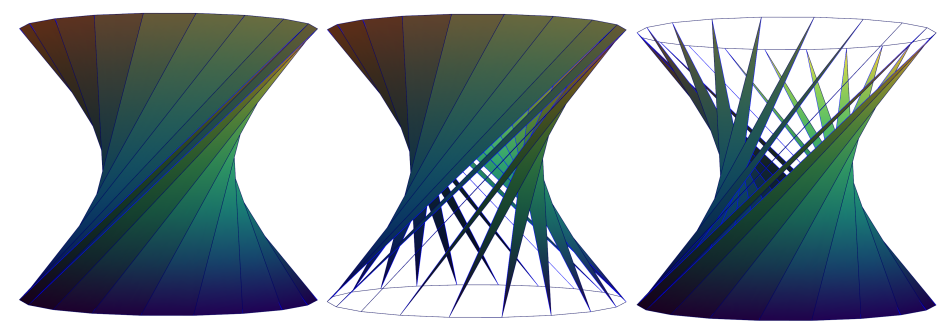

Fig. 1. Fejes Tóth's triangulation of the one-sheeted hyperboloid.

particular case we can explicitly calculate $d_{H}\left(\Sigma, T_{m}\right)$ and easily show that this triangulation is not optimal.

All calculations were performed by hand and verified using Mathematica, while producing the figures.

\section{The triangulation of the hyperboloid}

We prove that for the triangulation of the one-sheeted hyperboloid (with two circles of equal size as boundary) suggested by Fejes Tóth we have $d_{H}\left(T_{m}, \Sigma\right) \sim 1 / m$.

We parametrize the hyperboloid (one sheet of the surface determined by the equation $x^{2}+y^{2}-z^{2}=1$ ) by

$$
\sigma(u, t)=\frac{u}{\sqrt{2}}\left(\begin{array}{c}
-\sin t \\
\cos t \\
1
\end{array}\right)+\left(\begin{array}{c}
\cos t \\
\sin t \\
0
\end{array}\right)
$$

The surface consists of a one-parameter family of rulings, one for each $t$, given by $u \mapsto \sigma(u, t)$. We shall assume that $u \in\left[-u_{\max }, u_{\max }\right]$, so that the two circles that form the boundary lie at a distance $u_{\max } / \sqrt{2}$ above and below the $x y$-plane. In our parametrization we take $t \in[0,2 \pi]$.

The vertices of the triangulation discussed by Fejes Tóth are equally distributed along the lower and upper boundary and placed such that for every vertex on the lower boundary there is a vertex on the upper boundary that lies on the same ruling as the vertex on the lower boundary. Fejes Tóth assumes that $m$ is even. The triangulation is characterized by the fact that these rulings connecting the vertices are edges of the triangulation.

The edges of the triangles in the triangulation fall into three different categories:

- rulings that lie on the surface, 


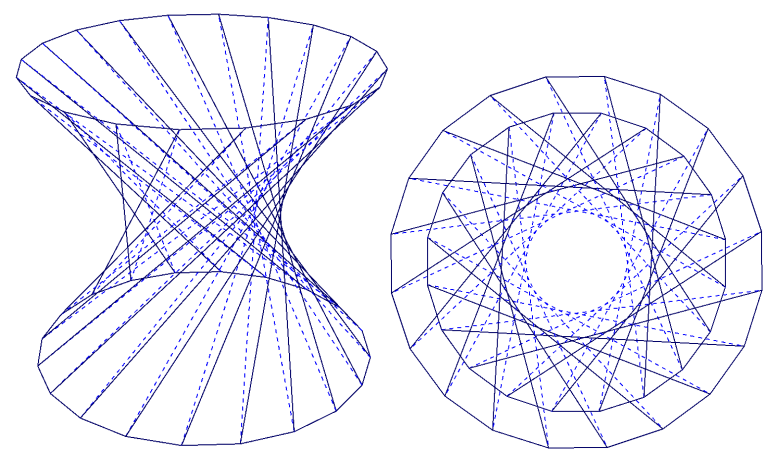

Fig. 2. The one-sheeted hyperboloid as wire model with the edges of the triangulation added.

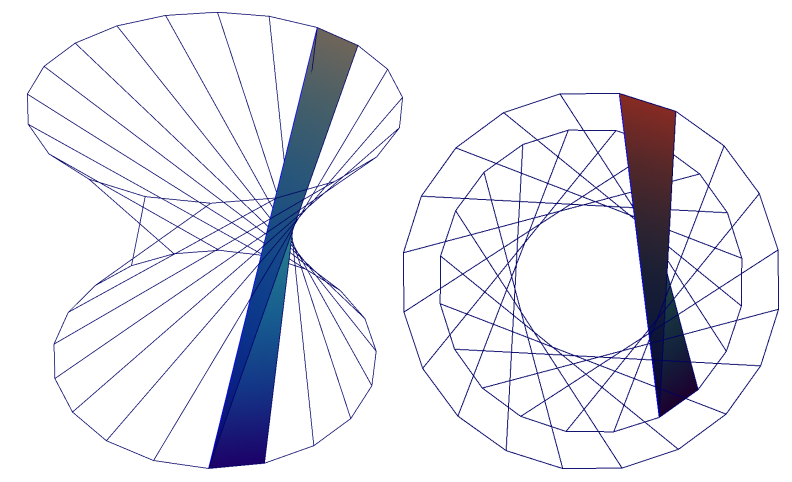

Fig. 3. The one-sheeted hyperboloid with two of the triangles of the triangulation inserted.

- edges of a regular $m / 2$-gon approximating the upper or lower boundary,

- edges that connect 'neighbouring' vertices on the upper and lower boundary.

Up to a rotation around the $z$-axis edges from the last category can be parametrized by

$$
\lambda \sigma\left(u_{\max }, 0\right)+(1-\lambda) \sigma\left(-u_{\max }, s\right)
$$

with $s=4 \pi / m$.

We now prove a lemma that refutes Fejes Tóth's claim that $d_{H}\left(T_{m}, \Sigma\right) \sim$ $1 / m^{2}$ for the triangulations described above:

LEMMA 2.1. For the sequence of triangulations $T_{m}$ of the one-sheeted hyperboloid $\Sigma$ bounded by two congruent circles suggested by Fejes Tóth, as 
described above, we have

$$
\lim _{m \rightarrow \infty} d_{H}\left(\Sigma, T_{m}\right) m=\sqrt{2} \pi u_{\max }
$$

Proof. To determine $d_{H}\left(\Sigma, T_{m}\right)$ we first give an upper bound. This bound is found by considering the triangulation and the surface restricted to horizontal planes. The hyperboloid restricted to a horizontal plane is a circle. The restriction of the triangulation is a (somewhat complicated) polygon, see Figure 4. The vertices of this polygon lie on the circle or are the restriction of an edge like (2), that is the intersection of a horizontal plane and the edge. For each horizontal plane we can determine the Hausdorff distance between the polygon and circle. This is straightforward because it is attained in the restriction of an edge like (2), due to the negative curvature of the surface. The maximum of all these pseudo-distances, defined below, bounds the Hausdorff distance between the hyperboloid and Fejes Tóth's triangulation. This maximum is attained for $z=0$, where the normal to the hyperboloid is horizontal so that here this pseudo-distance coincides with the Hausdorff distance between the hyperboloid and Fejes Tóth's triangulation.

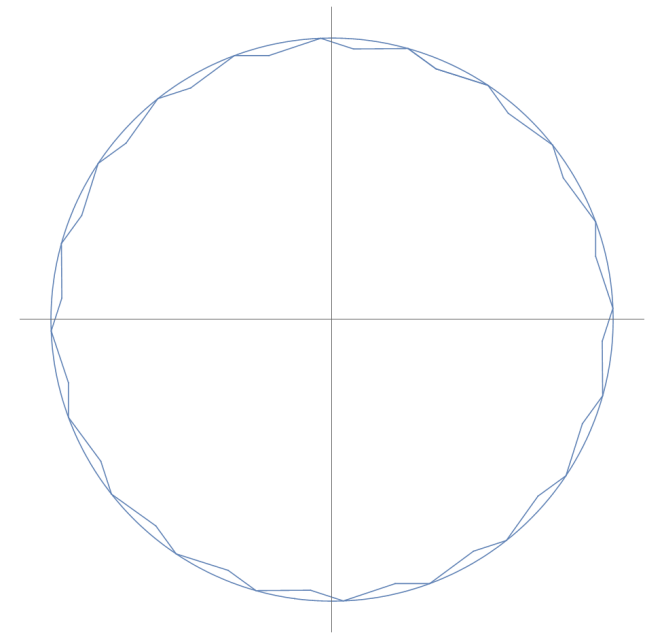

Fig. 4. A typical restriction of $T_{m}$ to a horizontal plane.

To find the Hausdorff distance between the polygon and circle, it is convenient to introduce the pseudometric

$$
d_{\mathrm{hor}}(v, w)=\sqrt{\left(v_{1}-w_{1}\right)^{2}+\left(v_{2}-w_{2}\right)^{2}},
$$

where $v=\left(v_{1}, v_{2}, v_{3}\right) \in \mathbb{R}^{3}$ and $w=\left(w_{1}, w_{2}, w_{3}\right) \in \mathbb{R}^{3}$. The difference between a metric space and a pseudometric space is that in case of the latter 
$d(x, y)=0$ does not imply $x=y$. Note that $d_{\text {hor }}(v, w) \leq|v-w|$, where $|\cdots|$ denotes the Euclidean norm. We are now able to calculate

$$
\begin{aligned}
d_{\text {hor }}\left(\lambda \sigma\left(u_{\max }, 0\right)\right. & \left.+(1-\lambda) \sigma\left(-u_{\max }, s\right), o\right) \\
& =\frac{\sqrt{2+(1-2 \lambda)^{2} u_{\max }^{2}}}{\sqrt{2}} \\
& -\frac{2 \lambda(\lambda-1) u_{\max }}{\sqrt{2+(1-2 \lambda)^{2} u_{\max }^{2}}} s+\mathcal{O}\left(s^{2}\right),
\end{aligned}
$$

where $o=(0,0,0)$. Note that $s=4 \pi / m$ and we are interested in the limit of $m$ tending to infinity. On the other hand the $z$-coordinate of the edge parametrized by $(2)$ is

$$
\frac{(2 \lambda-1) u_{\max }}{\sqrt{2}}
$$

so the hyperboloid restricted to the plane characterized by the coordinate is a circle with radius

$$
\frac{\sqrt{2+(1-2 \lambda)^{2} u_{\max }^{2}}}{\sqrt{2}}
$$

this radius is exactly the constant term in (3). This implies that the supremum of remaining terms in (3) is an upper bound on the Hausdorff distance

$$
\sup _{\lambda \in[0,1]}\left|\frac{2 \lambda(\lambda-1) u_{\max }}{\sqrt{2+(1-2 \lambda)^{2} u_{\max }^{2}}} s\right|+\mathcal{O}\left(s^{2}\right)=\frac{u_{\max }}{2 \sqrt{2}} s+\mathcal{O}\left(s^{2}\right) .
$$

The supremum of the expression on the left hand side of (4) is attained at $\lambda=\frac{1}{2}$, this can be seen by inspecting the nominator and denominator individually. At $\lambda=\frac{1}{2}$, we have that $z=0$ and the normal to the hyperboloid is horizontal for $z=0$. This means that the point $p$ where the supremum is attained and the point $p^{\prime}$ on the surface that is closest to $p$ both lie in the $z=0$ plane, by [2, Theorem 4.8.12]. This in turn implies that the right hand side of (4) equals the Hausdorff distance. We therefore find that

$$
\lim _{m \rightarrow \infty} d_{H}\left(\Sigma, T_{m}\right) m=\sqrt{2} \pi u_{\max }
$$

Lemma 2.1 contradicts the assertion of Fejes Tóth.

Acknowledgements. The authors are greatly indebted to Dror Atariah, Günther Rote and John Sullivan for discussion and suggestions. The authors also thank Jean-Daniel Boissonnat, Ramsay Dyer, David de Laat and Rien 
van de Weijgaert for discussion. This work has been supported in part by the European Union's Seventh Framework Programme for Research of the European Commission, under FET-Open grant number 255827 (CGL Computational Geometry Learning) and ERC Grant Agreement number 339025 GUDHI (Algorithmic Foundations of Geometry Understanding in Higher Dimensions), the European Union's Horizon 2020 research and innovation programme under the Marie Skłodowska-Curie grant agreement number 754411, and the Austrian Science Fund (FWF): Z00342_N31.

\section{REFERENCES}

[1] Do Carmo, M. P., Differential Geometry of Curves and Surfaces, Prentice-Hall, 1976.

[2] Federer, H., Curvature measures, Transactions of the America mathematical Society, 93 (1959), 418-491.

[3] Fejes Tóth, L., Lagerungen in der Ebene, auf der Kugel und im Raum, Berlin, Göttingen, Heidelberg: Springer, 1953.

[4] O’Neill, B., Elementary Differential Geometry, Elsevier, 2006.

[5] SpIvak, M., A comprehensive introduction to differential geometry: Volume I to $V$, Publish or Perish, 1999.

Open Access statement. This is an open-access article distributed under the terms of the Creative Commons Attribution-NonCommercial 4.0 International License (https://creativecommons.org/licenses/by-nc/4.0/), which permits unrestricted use, distribution, and reproduction in any medium for non-commercial purposes, provided the original author and source are credited, a link to the CC License is provided, and changes - if any - are indicated. 